\title{
A priori investigation of PDF-modeling assumptions for a turbulent swirling bluff body flame ('SM1')
}

\author{
R. De Meester ${ }^{\mathrm{a}}$, B. Naud ${ }^{\mathrm{b}}$, B. Merci ${ }^{\mathrm{a}}$ \\ ${ }^{a}$ Department of Mechanics of Flow, Heat and Combustion, Ghent University, \\ St-Pietersnieuwstraat 41, 9000 Gent, Belgium, reni.demeester@ugent.be \\ ${ }^{b}$ Modeling and Numerical Simulation Group, Energy Department, Ciemat, Avda. \\ Complutense 40,28040 Madrid, Spain, bertrand.naud@ciemat.es
}

Keywords: progress variable, PDF modeling, statistical independence, swirling flame

\section{Introduction}

The present study deals with a direct statistical analysis of experimental scatter data for the swirling non-premixed methane/air bluff-body flame 'SM1' [1-6], in the context of the popular non-premixed combustion modeling concept of mixture fraction and progress variable. For the flame studied, the scatter data in composition space, taken at fixed locations in physical space, is similar to what is observed in jet type flames with a substantial amount of local exinction, such as Sandia Flames E and F [7-9] in that there is strong deviation from steady-flamelet type lines in composition space (corresponding to little or no local extinction). A study on the experimental data of Sandia Flames D - F as discussed in [10] led to the formulation of the double conditioned CMC approach, using sensible enthalpy as progress variable. The latter is a measure for the deviation from the mentioned flamelet type lines or, equivalently, for the amount of local extinction. However, flame SM1 is different from jet type flames in that transported PDF simulations indicate that, close to the burner inlet, the deviations from flamelet type lines in composition space are not due to slower chemistry, caused by turbulence-chemistry interaction, but rather due to 'large scale' mixing of hot combustion products with air or fuel in the recirculation region behind the bluff body [11]. This motivates the present work. The a priori study on experimental data for SM1 reveals that using a mixture fraction - progress variable modeling approach in a RANS framework can lead to potentially 
large errors in the mean reaction rate. Different definitions of the progress variable and different shapes of the presumed joint PDF (product of marginal PDFs, using the assumption of statistical independence), are addressed.

\section{Progress variable definitions and presumed-PDF modeling as- sumptions}

In turbulent non-premixed combustion modeling, the PDF (probability density function) of mixture fraction $Z$ models the effect of turbulence on chemistry. In a reduced scalar approach (e.g. FGM [12], FPI [13], REDIM [14] and $\mathrm{ADF}_{-} \mathrm{PCM}_{\chi}$ [15], flamelet - progress variable (FPV) [16]), the 'progress' of the reaction is modeled by e.g. a 'progress variable' ( $c$ [15-17] or sensible enthalpy [10]) or a 'reaction progress parameter' $\lambda$ [18] [in [15], a third additional property, the scalar dissipation rate $\chi$, is also considered]. In the literature, many definitions appear for the progress variable, including: reduced temperature [18], sensible enthalpy [10] or a linear combination of species mass fractions $[16,17,19-21]$. In the present study, we refer to the latter with the symbol $Y_{c}$ and choose $Y_{c}=Y_{\mathrm{CO}_{2}}$. The definition $Y_{c}=Y_{\mathrm{CO}_{2}}+Y_{\mathrm{CO}}[19,22]$ leads to a better monotonicity of temperature and species in regions where $\mathrm{CO}_{2}$ decomposes into $\mathrm{CO}$, but this does not affect the observations in the study at hand (not shown).

Unless a transported PDF approach [23] is adopted, assumptions are commonly invoked on the shape of the PDF and on statistical (in)dependence of $Z$ and $c$ or $Z$ and $\lambda$. For instance, presumed PDF assumptions are introduced in the RANS modeling frameworks used in [19, 24]. The use of a progress variable in the context of presumed PDF modeling is mainly based on two assumptions: (i) statistical independence between $Y_{c}$ and $Z$, and (ii) modeling of the marginal PDFs of $Z$ and $Y_{c}$ based on their mean and variance.

Marginal PDF shapes. The probability density function (PDF) of $Z$ at point $\boldsymbol{x}$ and time $t, P(Z ; \boldsymbol{x}, t)$, is often approximated by a $\beta$-distribution based on the mean mixture fraction $\widetilde{Z}(\boldsymbol{x}, t)$ and mixture fraction variance $\widetilde{Z^{\prime \prime 2}}(\boldsymbol{x}, t)[25]$. For $P(c ; \boldsymbol{x}, t)$, and $P(\lambda ; \boldsymbol{x}, t)$ different shapes have been used. Although recently the 'statistically most likely distribution' has been introduced [20, 21], $\delta$ - and $\beta$-functions are still the most commonly used. In order to use a $\beta$ function in (ii), the progress variable must have the same range of values everywhere. Therefore, it is advantageous to normalize the progress variable such that the range becomes $[0-1]([26])$. Normalization of $Y_{c}$ can also help 
in satisfying assumption (i) [19, 24]. In [19, 24, 26], $Y_{c}(Z)$ is normalized with the equilibrium value $Y_{c, e q}(Z)$. Here, we normalize the progress variable $Y_{c}(Z)$ with $Y_{\mathrm{CO}_{2}}^{(\mathrm{fs})}(Z)$, the value on the flame sheet (Burke-Schumann model) going through $Y_{\mathrm{CO}_{2}}^{(\mathrm{fs})}\left(Z_{\mathrm{st}}\right)=0.1513$ at $Z_{\mathrm{st}}=0.054$ :

$$
c\left(Z, Y_{c}\right) \equiv Y_{c} / Y_{c}^{(\mathrm{fs})}(Z)=Y_{\mathrm{CO}_{2}} / Y_{C O_{2}}^{(\mathrm{fs})}(Z) .
$$

Whether the normalization is based on equilibrium values $Y_{c, e q}(Z)$ or flame sheet values $Y_{\mathrm{CO}_{2}}^{(\mathrm{fs})}(Z)$ is not essential for the study at hand. It merely affects the absolute values of $c(Z)$ (especially at the rich side), not the global observations.

Joint PDF and statistical (in)dependence. Considering the joint PDF, statistical independence of the variables is usually assumed: $P(Z, c)=P(Z) \cdot P(c)$ or $P(Z, \lambda)=P(Z) . P(\lambda)$, assumption (i). The general joint PDF definition involves a conditional PDF, though:

$$
P(Z, c ; \boldsymbol{x}, t)=P(Z ; \boldsymbol{x}, t) \cdot P(c \mid Z ; \boldsymbol{x}, t) .
$$

Comparison of conditional PDFs for different values of $Z$ thus indicates to what extent assumption (i) of statistical independence prevails. Figure 2 reveals that the normalization (1) leads to plateaus of constant $c$-values for non-premixed flamelets ${ }^{1}$ at the lean side and at the rich side (since the corresponding $Y_{\mathrm{CO}_{2}}$ profiles have shapes similar to the flame sheet). Only around stoichiometry there is a non-linear relationship between $c$ and $Z$. A limited number of measurements reveal $Y_{\mathrm{CO}_{2}}$ values, higher than $Y_{\mathrm{CO}_{2}}^{(\mathrm{fs})}(Z)$. In the normalization procedure, the value of $c$ for these points has been clipped to 1. They could also have been ignored in our analysis, if interpreted as 'outliers'. The clipping does not affect the global analysis at hand, given the fact that the number of points clipped is negligible in the amount of experimental data.

In [18], the 'reaction progress parameter' $\lambda$ quantifies the reaction 'progress':

$$
\lambda\left(Z, Y_{c}\right) \equiv\left\{Y_{c}^{(\text {stoich })}\right\}_{\text {flamelet ' } \mathrm{F} \text { ' }} \text { such that }\left(Z, Y_{c}\right) \text { is on flamelet ' } \mathrm{F} \text { '. }
$$

\footnotetext{
${ }^{1}$ The steady non-premixed flamelets represented in Figure 2 were calculated in the axisymmetric opposed-flow configuration, with Warnatz mechanism [27], assuming unity Lewis number, for different strain rates $\left(a=100,320,392 s^{-1}\right)$.
} 
In this definition, all points in $\left(Z, Y_{c}\right)$ or $(Z, c)$ space are assumed to lie on (stable or unstable) steady non-premixed flamelets and $\lambda$ denotes the value of the (traditional) progress variable $Y_{c}$ at stoichiometry on that steady nonpremixed flamelet. As long as the real flame structure corresponds to a collection of steady non-premixed flamelets, $\lambda$ is constant along each flamelet and as such becomes statistically independent of $Z$. In [28], still another progress variable is defined, based on the enthalpy of formation integrated over $Z$-space, making it statistically independent of $Z$.

Both definitions of the progress variable $c$ and of the reaction progress parameter $\lambda$ are useful with respect to condition (i) for presumed PDF modeling of flame SM1 if the composition in $\left(Z, Y_{\mathrm{CO}_{2}}\right)$ space mainly corresponds to steady turbulent non-premixed flamelets. It is shown in Section 4 that introducing such progress variables in a priori studies of the experimental data helps to identify steady non-pemixed flamelet structures.

Progress variable transport equation. With respect to $c$ and $\lambda$, the definitions of normalized progress variables or reaction progress parameters are useful in order to satisfy (i) and (ii) in the context of presumed PDF modeling. However, at some point the modeling of the mean or filtered value, and possibly the variance, of $c$ or $\lambda$ is required. The transport equations for these quantities contain non-negligible terms that are harder to model than the corresponding terms in the standard transport equations for $Y_{c}[21,29]$. In [21], in the context of LES, solving the transport equation for $\lambda$ is avoided by assuming a 'statistically most likely distribution' for $P(\lambda)$. In $[19,24]$, in the context of RANS simulations, an assumption is formulated for the mean progress variable (i.e. the first moment of the $\mathrm{PDF}$ ): $\overline{c \mid Z}=\bar{c}$. This assumption is less stringent than complete statistical independence, $P(c \mid Z)=$ $P(c)$, while solving the transport equation for the mean progress variable $\bar{c}$ is avoided. Instead, the transport equation for the mean non-normalized progress variable $\widetilde{Y}_{c}$ is solved and $\bar{c}$ is reconstructed afterwards from $\widetilde{Y}_{c}$. It is illustrated below, though, that for the flame at hand the assumption $\overline{c \mid Z}=\bar{c}$ is questionable.

A priori study. The study at hand concerns a direct analysis of experimental data in the sense of RANS modeling. As mentioned, the scatter in the experimental data of flame SM1 is primarily caused by 'large scale' mixing of hot combustion products with air or fuel in the recirculation region behind the bluff body [11], not by local extinction (or lack in 'progress' of the 
reaction). As such, the case resembles 'multiple stream mixing'[30], where the combustion products act as third stream. It is illustrated below that commonly made assumptions in RANS frameworks do not prevail: $Z$ and $c$ or $Z$ and $\lambda$ are not statistically independent; and $\overline{c \mid Z} \neq \bar{c}$.

\section{Marginal PDFs}
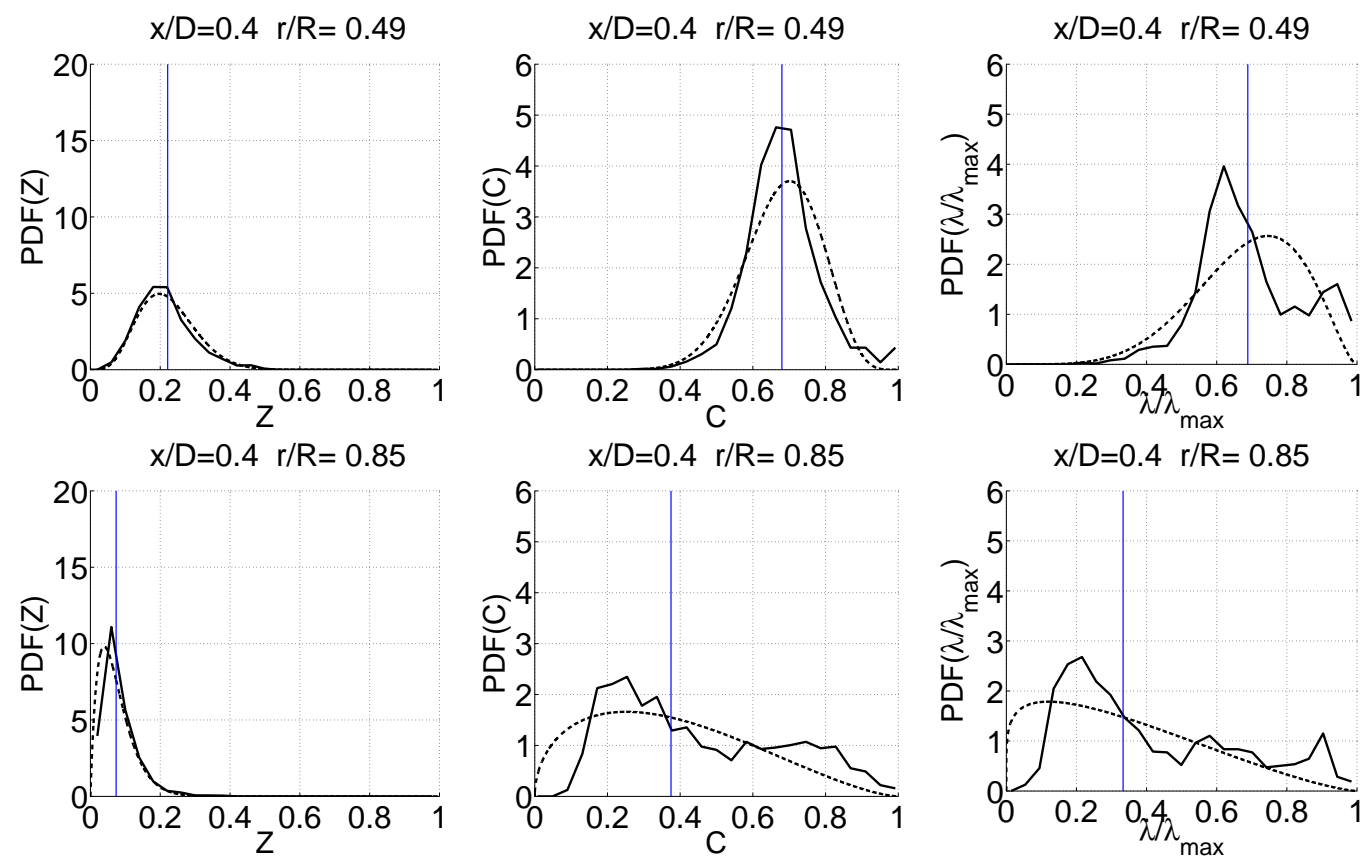

Figure 1: Experimental PDF (full line) and corresponding $\beta$-PDF (dashed line) at $x / D=$ $0.4(r / R=0.45$ and $r / R=0.85)$ for $Z$ (left), $c$ (middle) and $\lambda$ (right). The vertical line indicates the mean value $(\delta-\mathrm{PDF})$.

Figure 1 shows the marginal PDFs of $Z, c$ and $\lambda$ as retrieved from the experimental data at $x / D=0.4$ (which is close to the bluff body, inside the recirculation region). The results at other axial locations are similar (not shown). The radial locations have been chosen close to the shear zone because there the mixing is the strongest and the largest differences are expected $(r$ is the distance from the symmetry axis and $R=D / 2$ ). In order to have a sufficient amount of samples in each bin, the experimental data has been divided for each scalar $(Z, c$ or $\lambda)$ into 25 equally sized bins. The analysis presented below has been confirmed to remain valid when the bins have only 
half the size. The experimental PDFs of $c$ and $\lambda$ include non-zero values at $c=1$ and $\lambda=1$ due to the clipping of experimental measurements above the flame sheet in $\left(Z, Y_{c}\right)$ space, but this is not relevant in the discussion at hand. [Note that $\lambda$ has been normalized with $\lambda_{\max }=Y_{C O_{2}}^{\max }\left(Z_{\text {stoich }}\right)=0.1513$ in order to make the range of all horizontal axes equal to [0-1].]

The corresponding $\beta$-PDFs, with the same values for mean and variance as retrieved from the experimental data, are shown to be good approximations for the marginal PDF of $Z$, while for $c$ and $\lambda$ the strong deviations observed at some locations indicate that the $\beta$-PDF assumption is not generally applicable for these variables. The vertical lines at the mean value represent the $\delta$-PDF. In general, the $\delta$-PDF assumption is clearly insufficient to model any of the marginal $Z, c$ and $\lambda$ PDFs. These observations are in line with [20]: higher order statistical information is needed to represent complex PDFs (highly skewed or bimodal PDFs).

We recall that the experimental data is treated here in a RANS modeling sense. The above observations do not give information about the validity of models for the shape of the marginal filtered density functions (FDF) in a LES context, i.e. on the modeling of subgrid fluctuations which are actually conditional statistics (conditioned on the resolved large scales).

\section{Statistical (in)dependence and flame structures}

Comparison of conditional PDFs. At a given location, if $Z$ and $c$ (or $Z$ and $\lambda$ ) are independent, the conditional $\operatorname{PDF} P(c \mid Z)$ (or $P(\lambda \mid Z)$ ) becomes identical to the marginal $\operatorname{PDF} P(c)$ (or $P(\lambda)$ ), for all $Z$-values. Figure 2 shows the conditional PDFs $P\left(Y_{\mathrm{CO}_{2}} \mid Z\right), P(c \mid Z)$ and $P(\lambda \mid Z)$ at two points in first recirculation zone behind the bluff body $(x / D=0.4)$ : in the shear zone where recirculated combustion products mix with air from the swirling annulus $(r / R=0.85)$ and in the inner recirculation zone $(r / R=0.49)$. A rich flamelet branch predominantly characterized by the mixing between combustion products at stoichiometry and pure fuel is observed at $r / R=$ 0.49, while a mixing line representing mixing of fresh air with rich combustion products [11], caused by the recirculation of combustion products, is observed at $r / R=0.85$.

At $r / R=0.49$, a large difference is observed between the conditional PDFs of $Y_{\mathrm{CO}_{2}}$ and those of $c$ or $\lambda$. The latter stay fairly constant as a function

of $Z$, indicating that $Z$ and $c$ or $Z$ and $\lambda$ are rather statistically independent, while this is clearly not the case for $Z$ and $Y_{\mathrm{CO}_{2}}$. The observed deviations 

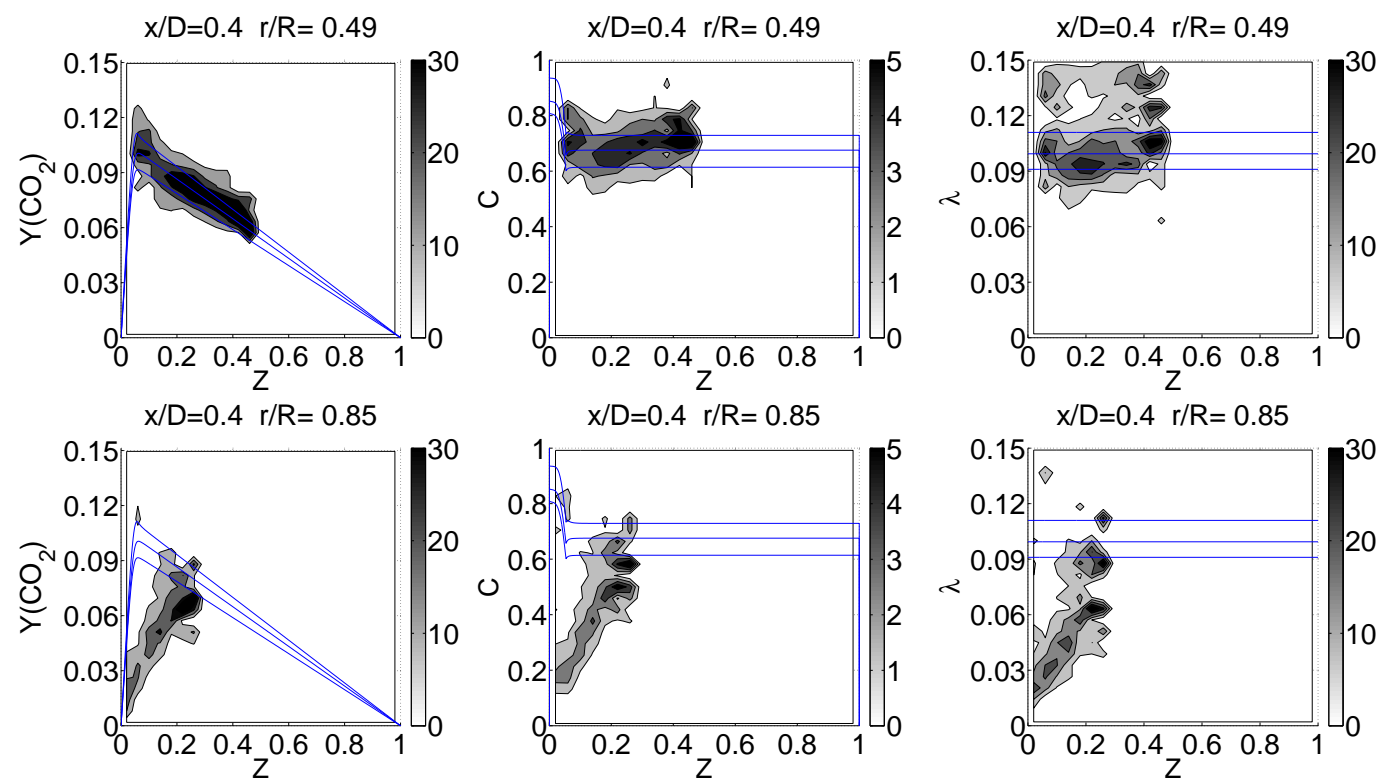

Figure 2: Conditional $Y_{\mathrm{CO}_{2}}{ }^{-}, c$ - and $\lambda$-PDF at $x / D=0.4$ at $r / R=0.49$ and $r / R=$ 0.85. Probability indicated with gray scale ranging from white (zero probability) to black (highest probability). Solid lines correspond to steady non-premixed flamelets.

from the fully burning flamelets can be explained by local extinction, i.e. by the combination of finite-rate chemistry and high local scalar dissipation. 'Extinguished fluid' at lower reaction progress can mix with extinguished fluid or with burning fluid from the lean and rich branches of the flamelet structure. In other words, local extinction 'creates' new boundary conditions for the local mixing problem [9], resulting in more spreading in composition space. In this case, we can expect a moderate impact on the assumptions $\overline{c \mid Z}=\bar{c}$ and $\overline{\lambda \mid Z}=\bar{\lambda}$. This was also seen for the SANDIA D flame [19], which only has a small amount of local extinction.

At $r / R=0.85$ (mixing line), the three conditional PDFs are similar, all indicating a linear dependence between $Z$ and the progress variable $\left(\mathrm{Y}_{\mathrm{CO}_{2}}\right.$, $c$ or $\lambda$ ). In this case, $\overline{c \mid Z} \neq \bar{c}$ and $\overline{\lambda \mid Z} \neq \bar{\lambda}$ as this mixing line differs too strongly from any steady non-premixed flamelet structure (horizontal line) or premixed flamelet structure (vertical line). Therefore none of the proposed definitions can ensure independence of $Z$ with the progress variable. Note that in this case, $Y_{\mathrm{CO}_{2}}, c$ and $\lambda$ do not really indicate the progress of reaction as it would in a reacting homogeneous mixture, but rather the 
mixing between fresh air and recirculated combustion products without any significant reaction (see also [11]). This recirculation zone 'creates' a third stream [30] of rich combustion products, resulting in a mixing of air with rich combustion products. This leads to deviation from the flamelet structure, in a confined, line shaped structure in composition space. In a RANS framework, where all mixing scales are modeled together, this results in $\overline{c \mid Z} \neq \bar{c}$ and $\overline{\lambda \mid Z} \neq \bar{\lambda}$.
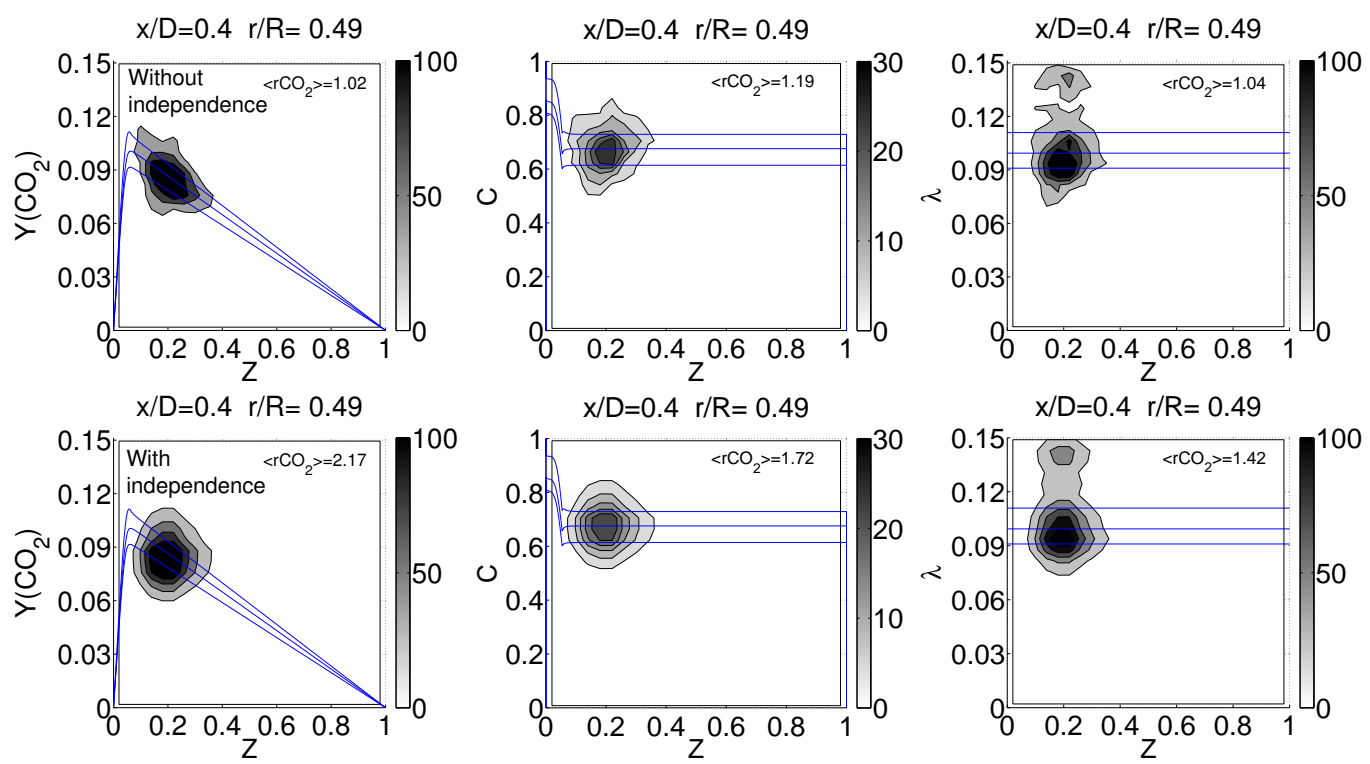

Figure 3: Joint PDFs at $x / D=0.4, r / R=0.49$. Top: experimental $Z-Y_{\mathrm{CO}_{2}}, Z-c$ and $Z-\lambda$ joint PDFs (no statistical independence assumed). Bottom: products of the experimental marginal PDFs, $P(Z) \cdot P\left(Y_{\mathrm{CO}_{2}}\right), P(Z) . P(c)$ and $P(Z) . P(\lambda)$ (i.e. statistical independence assumed). Probability indicated with gray scale ranging from white (zero probability) to black (highest probability.) $\left\langle r \mathrm{CO}_{2}\right\rangle$ : corresponding mean production rate of $\mathrm{Y}_{\mathrm{CO}_{2}}$, calculated with REDIM table.

Comparison of joint PDFs and mean reaction rate. The above observations on the assumption of statistical independence are confirmed in Figures 3 and 4, where the experimental joint PDFs are compared to the products of the experimental marginal PDFs at $x / D=0.4$. Similar results are obtained at other axial locations (not shown). The comparison is quantified by reporting the value for the mean production rate of $Y_{\mathrm{CO}_{2}},\left\langle r C \mathrm{O}_{2}\left(Z, \mathrm{Y}_{\mathrm{CO}_{2}}\right)\right\rangle$, as calculated from a REDIM table [14]. [Note that, for the different definitions of 

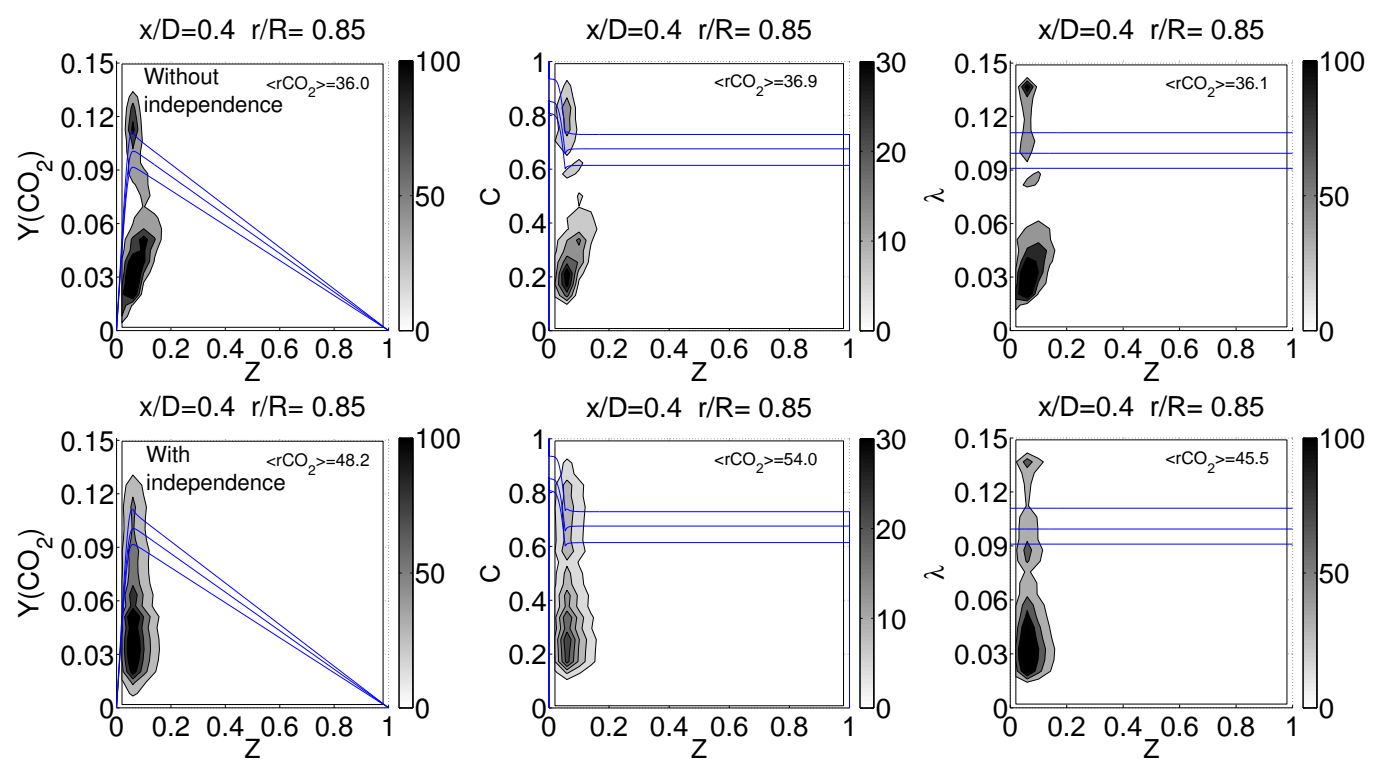

Figure 4: Joint PDFs at $x / D=0.4, r / R=0.85$. Legend: see Fig. 3 .

the progress variable, $\left\langle r \mathrm{CO}_{2}\right\rangle$ as calculated without the assumption of independence, i.e. the numbers in the top rows, should be the same. The small differences observed are due to discretisation errors.]

In Figure 3, we see that the error made on the mean reaction rate due to the assumption of statistical independence is reduced when considering the normalized progress variable $c$ or the reaction progress parameter $\lambda$. Qualitatively, we can see that the joint PDFs (with and without statistical independence) are indeed more similar when considering $c$ and $\lambda$, than when considering $Y_{\mathrm{CO}_{2}}$. However, Figure 4 shows that at point $r / R=0.85$, where the mixing line in composition space is observed, the introduction of $c$ or $\lambda$ does not help to reduce the error on the mean reaction rate when assuming statistical independence. The error remains of the same order as when considering $\mathrm{Y}_{\mathrm{CO}_{2}}$.

In this a priori study based on experimental data we could illustrate how the special flow feature of this bluff-body stabilized flame (the mixing between fresh air and recirculated combustion products above the bluff body) makes invalid the assumptions usually made in presumed PDF modeling based on a mixture fraction and a progress variable in a RANS framework. 


\section{Acknowledgements}

We would like to thank the originators of the experimental data for making it freely available on the internet [6]. This work is funded by the Special Research Fund of Ghent University under project BOF07/DOC/210, and also supported by the Comunidad de Madrid through Project HYSYCOMB P2009/ENE-1597 and by the Spanish Ministry of Economy and Competitiveness under Projects ENE2008-06515-C04-02 and CSD2010-00011.

\section{References}

[1] P.A.M. Kalt, Y.M. Al-Abdeli, A.R. Masri, R.S. Barlow, Proceedings of the Combustion Institute 29 (2003) 1913-1919.

[2] Y.M. Al-Abdeli, A.R. Masri, Experimental Thermal and Fluid Science 27 (5) (2003) 655-665.

[3] A.R. Masri, P.A.M. Kalt, R.S. Barlow, Combustion and Flame 137 (1-2) (2004) 1-37.

[4] Y.A. Al-Abdeli, A.R. Masri, G.R. Marquez, S.H. Starner, Combustion and Flame 146 (1-2) (2006) 200-214.

[5] A.R. Masri, P.A.M. Kalt, Y.M. Al-Abdeli, R.S. Barlow, Combustion Theory and Modelling 11 (5) (2007) 653-673.

[6] http://sydney.edu.au/engineering/aeromech/thermofluids/swirl.htm

[7] R.S. Barlow, Web site for the International Workshop on Measurement and Computation of Turbulent Non-premixed Flames (TNF), http://www.ca.sandia.gov/TNF/, 1996.

[8] A.R. Masri, R.W. Dibble, R.S. Barlow, Progress in Energy and Combustion Science 22 (4) (1996) 307-362.

[9] R.S. Barlow, J.H. Frank, Proceedings of the Combustion Institute 27 (1998) 1087-1095.

[10] A. Kronenburg and M. Kostka, Combustion and Flame 143 (2005) 342356 
[11] R. De Meester, B. Naud, U. Maas and B. Merci, Combustion and Flame 159 (7) (2012) 2415-2429

[12] J.A. van Oijen, L.P.H. de Goey, Combustion Science and Technology 161 (2000) 113-137.

[13] O. Gicquel, N. Darabiha, D. Thevenin, Proceedings of the Combustion Institute 28 (2000) 1901-1908.

[14] V. Bykov, U. Maas, Combustion Theory and Modelling 11 (6) (2007) 839-862.

[15] J.-B. Michel, O. Colin, D. Veynante, Combustion and Flame 152 (2008) 80-99.

[16] C.D. Pierce, P. Moin, Journal of Fluid Mechanics 504 (2004) 73-97.

[17] P. Domingo, L. Vervisch, K. Bray, Combustion Theory and Modelling 6 (2002) 529-551.

[18] M. Ihme, C.M. Cha, H. Pitsch, Proceedings of the Combustion Institute 30 (2005) 793-800.

[19] L. Vervisch, R. Hauguel, P. Domingo, M. Rullaud, Journal of Turbulence 5 (2004) 4.

[20] M. Ihme, H. Pitsch, Combustion and Flame 155 (1-2) (2008) 70-89.

[21] M. Ihme, H. Pitsch, Combustion and Flame 155 (1-2) (2008) 90-107.

[22] B. Fiorina, R. Baron, O. Gicquel, D. Thevenin, S. Carpentier, N. Darabiha Combustion Theory and Modelling 7 (3) (2003) 449-470.

[23] S.B. Pope, Progress in Energy and Combustion Science 11 (2) (1985) 119-192.

[24] J.-B. Michel, O. Colin, C. Angelberger, D. Veynante, Combustion and Flame 156 (2009) 1318-1331.

[25] N. Peters, Turbulent Combustion, Cambridge University Press, Cambridge, 2000. 
[26] R. Landenfeld, A. Sadiki, J. Janicka, Flow, Turbulence and Combustion 68 (2002) 111-135.

[27] J.Warnatz, U.Maas, R.W.Dibble, Combustion physical and chemical fundamentals, modeling and simulation, experiments, pollutant formation, Springer, Berlin, 2006.

[28] H. Lehtiniemi, F. Mauss, M. Balthasar, I. Magnusson, Combustion Science and Technology 178 (2006) 1977-1997.

[29] K. Bray, P. Domingo, L. Vervisch, Combustion and Flame 141 (2005) 431-437.

[30] J. Floyd, A.M. Kempf, A. Kronenburg, R.H. Ram Combustion Theory and Modelling 13 (4) (2007) 559-588. 